\title{
INVESTIGACIONES HISTÓRICO-ARQUEOLÓGICAS EN EL ANTIGUO Claustro del noviciado, hoy Casa de la Columna, del convento de Santo Domingo de Lima
}

\author{
Antonio Coello Rodrigueza
}

\begin{abstract}
Resumen
El siguiente articulo presenta un resumen de las excavaciones arqueológicas realizadas al interior del antiguo claustro del noviciado, el mismo que fuera parte original del convento de Santo Domingo de Lima. En la actualidad, este claustro es conocido como la "Casa de la Columna» y se ubica en el actual jirón Conde de Superunda, cuadra dos. Estas excavaciones formaron parte de un proyecto multidisciplinario, el cual tenía como objetivos la restauración del claustro, así como la portada interior; en otros casos, se consolidarian otras estructuras originales que igualmente eran parte del antiguo Claustro del Noviciado (nos referimos a la arquería misma del claustro). En la actualidad, la Casa de la Columna es una gran casa de vecindad que, en el Perú, se denomina "callejón», en la cual moran más de 200 habitantes, los cuales han tugurizado por completo el antiguo recinto religioso. Luego de que el claustro del noviciado fuera convertido en casa de vecindad, hacia la segunda mitad del siglo XIX, albergó en sus antiguas celdas — donde vivían los sacerdotes - a varias personas civiles que practicaban diversos oficios, incluso, a familias enteras, las cuales poco a poco fueron alterando la fisonomía original del lugar. A la vez, el lugar empezó a soportar la cotidianidad de una nueva sociedad y quedaron como restos de esta nueva vida elementos ajenos a una vida religiosa.
\end{abstract}

Palabras clave: arqueología histórica, convento de Santo Domingo de Lima, Lima, Casa de la Columna, vida cotidiana en la Lima del siglo XIX

Abstract

HISTORICAL-ARCHAEOLOGY IN THE ANCIENT CLAUSTRO DEL NOVICIADO, PRESENT-DAY CASA DE LA COLUMNA, OF SANTO DOMINGO CONVENT IN LIMA

This article is a summary of the archaeological excavations carried out inside the old cloister of the "Convent of Santo Domingo de Lima", known today as "La Casa de la Columna", and located on the second block of the Calle Conde Superunda. These excavations were part of a multidisciplinary project whose main objective was to restore, and in some cases consolidate, the original structures that were part of the old convent (i.e., the cloister's archway and entrance). Today La Casa de la Columna is a densely populated habitation with more than 200 residents. After this cloister was turned into a tenement house towards the second half of century XIX, the former priests' cells came to house several people including whole families who altered the place's original appearance little by little over time. These changes involve the addition of elements characteristic of the lifestyle of the inhabitants' new society, which are quite different from those of the place's previous religious function

Keywords: historical archaeology, convent of Santo Domingo de Lima, Lima, Casa de la Columna, daily life in Lima during 19th century

\footnotetext{
a Universidad Nacional Mayor de San Marcos

Correo electrónico: sequilao@yahoo.com
} 


\section{Lima y los primeros ańos del convento dominico}

Al fundarse Lima hacia 1535 , se instalaron, inmediatamente a la par, las diversas órdenes religiosas que vinieron junto al conquistador español: mercedarios, dominicos, franciscanos. De estos, los dominicos estuvieron en una situación muy privilegiada, debido a que ellos se adjudicaron cerca de cuatro manzanas enteras para construir su futuro convento de Nuestra Seńora del Rosario.

Para entender los primeros ańos fundacionales del convento dominico, debemos utilizar dos fuentes muy tempranas. La primera es la dejada por el cronista dominico Juan Meléndez (Fig.1), quien indica, respecto al antiguo convento de Nuestra Señora del Rosario, que la iglesia medía 84 varas de largo (65 metros aproximadamente), 36 de ancho (29 metros) y 18 de alto (cerca de 14 metros). Se dividía en tres naves. La central medía 15 varas de ancho (aproximadamente, 12 metros) y estaba formada por tres partes: capilla mayor; el cuerpo, que llaman de iglesia; y la gran pieza de coro. La capilla mayor comprende el presbiterio, el crucero y dos capillas colaterales ${ }^{1}$.

En cuanto al antiguo Claustro del Noviciado, hoy patio de la Casa de la Columna, sitio donde realizamos nuestra investigación, nos sirvió de mucho lo descrito por el cronista Dominico Juan Meléndez, quien afirma que fue «el segundo Claustro algo menor que el primero pero de la misma fábrica: tiene como el principal su fuente de agua en el medio: y del por distintos tránsitos, se pasa al Noviciado, que puede ser convento de por sí, con capilla en que está colocado el Santísimo Sacramento, Claustros, fuentes y dormitorios, y a otros 3 Claustros de la misma simetría, que los primeros dispuestos con lindo orden, y con ser tanto los frailes, que moran en el Convento, que es lo ordinario pasar de 250, es tan crecido el número de celdas, que no solo hay para todos si no que sobran varias celdas, para muchos más» (1681: 61, el énfasis es nuestro).

Tal como puede haberse leído, el Claustro del Noviciado presentaba una capilla; una fuente o pileta al centro, por donde discurría agua; y diversas celdas, luego convertidas en recintos habitacionales, en donde se cobijaron los diferentes inquilinos de la Casa de la Columna. Esta cita corroboró la información obtenida en campo. De este modo, pudimos contrastar dos tipos de fuentes, la histórica y la arqueológica, para tener la certeza de lo que íbamos hallando durante el proceso de excavación, sus usos y nuevos cambios.

Por otro lado, una segunda fuente conocida y muy apreciada es la de Bernabé Cobo, quien en su obra Historia de la fundación de Lima, escrita hacia 1613, nos manifiesta la rica opulencia dominica. Cobo la describe de la siguiente manera «La Iglesia es muy grande y de costosa fábrica; de una nave con dos órdenes de capillas por los lados; estas son de bóvedas curiosamente labradas, y la nave de en medio cubierta de madera y luzeria [sic] curiosa; la capilla mayor es de bóveda y para tan grande iglesia es tenida por pequeña» (1882: 259).

En cuanto al interior del convento dominico, tema de nuestro interés, Cobo nos comenta: «El Claustro principal es el más bien adornado de todos los de este reino; tiene las paredes y pilares bajos por más de estado y medio desde el suelo cubiertos de azulejos de variados y curiosos labores, los cuales se trajeron con gran costo de España [...] en medio de este patio está una hermosa fuente de piedra; sin este tiene el convento tres o cuatro patios menores, muchas celdas altas y bajas con todas las oficinas muy cumplidas; edificio todo de tan buena fabrica» (Cobo 1882: libro III, cap. III).

Ambas fuentes tempranas nos hacen imaginar lo ostentoso y magnífico del convento de Nuestra Señora del Rosario; sin embargo, este apogeo será mermado posteriormente, luego de dos grandes terremotos: el primero fue el de 1687; y el segundo, de 1748. Sendos terremotos ocasionaron grandes cambios a nivel estructural y funcional al convento dominico. El 17 de junio de 1687, Lima soportó un gran terremoto, el cual afectó enormemente la aún pequeña ciudad de Lima; el convento dominico no estuvo exento de esta catástrofe. Como prueba de ello, podemos enterarnos de los daños ocasionados a través de una fuente de primer orden, como es la de Domingo Angulo: «El convento de Santo Domingo, la iglesia, capillas, altar y demás oficinas esta caída, demolido y arruinado, abiertas brechas por diferentes partes, el coro alto y bajo y sus bóvedas hundidas, y la torre caída al suelo, de donde dijo el padre Superior Fray Manuel de 


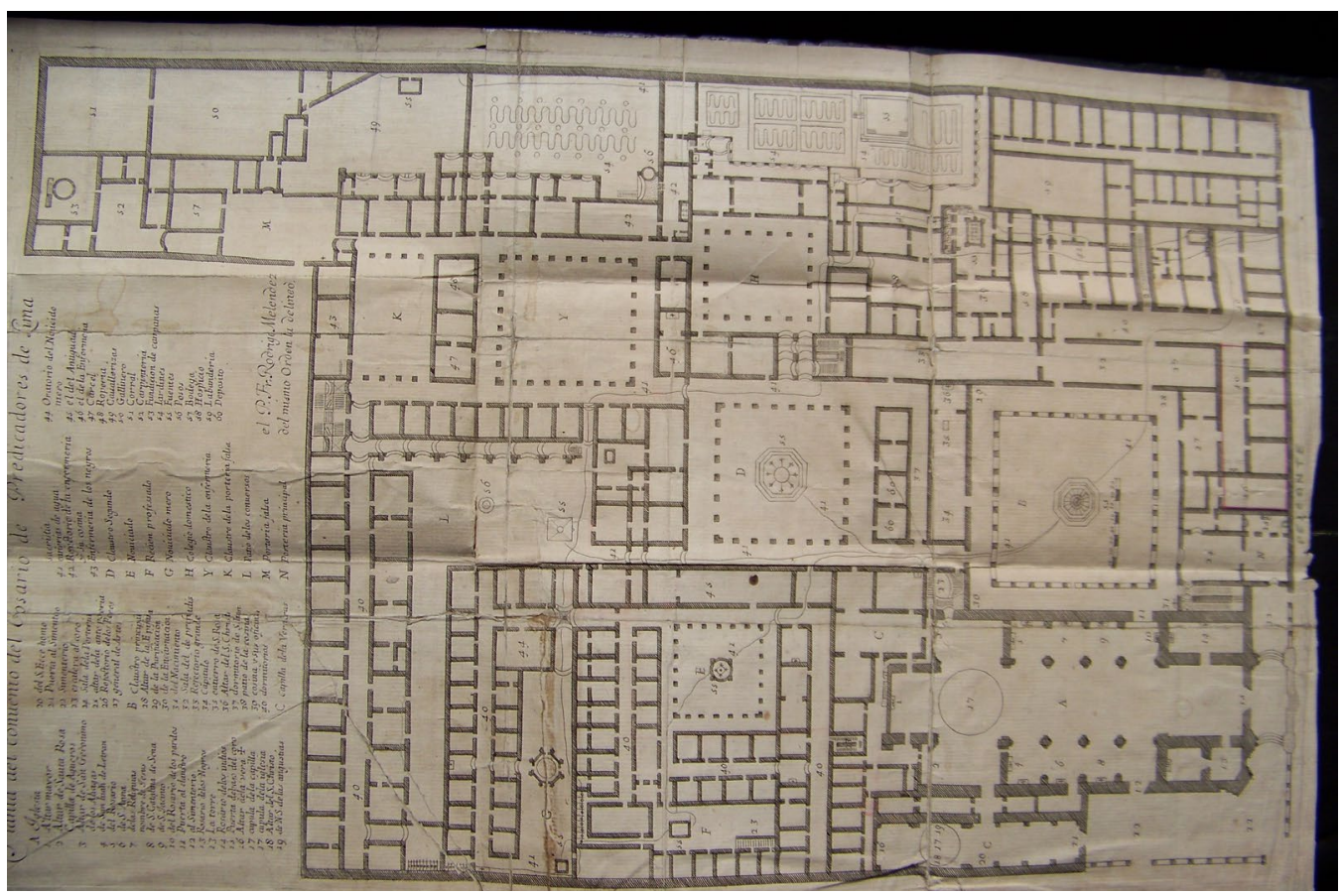

Figura 1. Plano del convento original de Santo Domingo levantado por Juan Meléndez hacia 1681.

Castro, habían sacado de sus ruinas 42 cuerpos muertos, y se juzgaba haber más; los claustros, cuatro altos y cuatro bajos, caídos y arruinados en tierra; librería, Colegio de San Juan Bautista, que está dentro de dicho convento, arruinado y sin forma, porque todo lo arraso el terremoto, con el noviciado, profesado, sacristía y demás oficinas, sin que quedase cosa alguna, si no es en la iglesia la capilla de Nuestra Señora del Rosario, que quedo ilesa» (1939: 30).

Este terremoto ocasionó una destrucción casi total a todo el convento, el cual tuvo que volver a levantarse, por decir lo menos, desde sus cimientos. Ello ocasionó daños a la capilla mayor de la iglesia, por lo que fue preciso derribarla. Asimismo, se reedificaron las capillas laterales, y se remplazó el material constructivo utilizado en las bóvedas — ladrillo y piedra - por una armazón de madera y caña. Posteriormente, otro terremoto — de mayores consideraciones- asolaría Lima y su principal puerto, El Callao. Nos referimos al sismo del 28 de octubre de 1746 (Ulloa y Juan 1748[1703-1776]: 42-43), el cual ocasionó pánico y destrucción total de Lima y de Callao. Según las fuentes escritas, perecieron en Lima no menos de 10.000 personas el mismo día. Este sismo igualmente afectó a todo el convento dominico, que, de la misma forma que el anterior terremoto del siglo pasado, volvería a alterar la arquitectura conventual. Sobre el famoso terremoto de 1746 que asoló Lima, puede consultarse la reciente publicación de Walker (2012), en la que se describen las consecuencias calamitosas, en la que quedó la ciudad de Lima.

$\mathrm{La}$ acentuada crisis financiera en la que se hallaba sumergido el convento de Santo Domingo iba envolviendo de la misma manera, poco a poco, a toda la ciudad. Ante este grave problema, el convento de Santo Domingo empezaría a alquilar y vender, en otros casos, parte de sus antiguos bienes y terrenos. Uno de los casos específicos fue el de la venta de uno de sus claustros, el cual sería vendido hacia 1790, luego del terremoto de 1746, para convertirse en una gran residencia, conocida desde entonces como la Casa de Superunda, ubicada en la calle Matavilela (Huertas y Flores Espinoza 1981). Con esta primera venta, los dominicos empezarían a ver poco a poco cómo disminuía su antiguo convento, pero, a cambio, obtendrían cuantiosas rentas que aliviarían sus arcas venidas a menos. 
Los dominicos, al igual que otras órdenes, poseedoras de diversos terrenos y edificaciones (fincas, casas, tiendas, pulperías, casas de vecindad y demás tipos de viviendas), supieron afrontar estos problemas, tal como lo ha señalado Luna (2002: 141-163) para el caso específico de la congregación de la Buenamuerte de la ciudad de Lima.

Como consecuencia de la independencia, el reciente creado Estado peruano sería centralista y secular, manejado por una «élite» local limeña. Estos nuevos aires independentistas ocasionarían entre otros cambios que las diversas órdenes religiosas empiecen a tener menores ingresos económicos. Asimismo, sus seguidores disminuirían enormemente, lo que ocasionaría que los amplios, pero ya vetustos claustros conventuales venidos a menos, empiecen a estar vacíos y sin la cantidad de gente a la que estaban acostumbrados. Ante tal problema, el clero religioso empezaría a arrendar y alquilar a terceros sus antiguas edificaciones para así poder obtener mayores ingresos económicos y solventar sus alicaídas arcas.

Sumado a estos daños, un tiro de gracia ahondará esta crisis: el decreto de supresión de los conventos de 1826, que afectó enormemente los terrenos del convento de Santo Domingo (Rohr 2005: 91).

\section{Siglo XIX, de convento a casa de vecindad}

Al llegar las primeras décadas del temprano siglo XIX, el panorama de Lima mostrará todavía el predominio de las órdenes religiosas de perfil urbano. Aún sobresalen las grandes edificaciones religiosas sobre una ciudad pequeñamente horizontal, pero no se observa ningún asomo de palacio, cárcel u otro tipo de edificación civil majestuosa.

Una vez instaurado y desarrollado un nuevo tipo de sociedad más laica, Santo Domingo, al igual que los otros conventos limeños, soportó mayores crisis económicas que mermaron aún más sus terrenos originales. De su rico pasado arquitectónico, solamente quedaron algunos pocos espacios, tal como nos lo dejó escrito el viajero francés Max Radiguet: «Ese convento posee varios patios, cuyo centro está ocupado por una fuente de bronce; todas están rodeadas por dos hileras superpuestas de claustros con arcadas cimbradas. Solo el primero de esos claustros, es decir el que está próximo a la Iglesia, es conservado con cuidado» (1971: 91, el énfasis es nuestro).

De la extensión original, los dominicos poco a poco fueron vendiendo en enfiteusis ${ }^{2}$ otros sectores de su convento. De este modo, parte del noviciado fue usado como refectorio y el resto - un claustro de cuatro por siete columnas, una capilla, un patio con arquería a un lado y unas celdas - fue utilizado como casa de vecindad con inquilinos provisionales.

El que fuera el original Claustro del Noviciado sería utilizado hacia 1830 y 1840 como cuartel de policía, y, a la vez, como casa de vecindad ${ }^{3}$. Luego, entre las décadas de 1850 y 1860, el comerciante Nicolás Rodrigo alquiló varios ambientes restantes de lo que había sido el noviciado «Cuarto por cuarto, juntó en seis compras diferentes las áreas que quedaban en el centro de la manzana, hasta obtener el conjunto que desde entonces fue denominado Casa de la Columna» (Rohr 2005: 96, el énfasis es nuestro). Es posible que el nombre se deba a una mención irónica del hecho de que, para adecuar el ingreso, fue necesario remover una columna de la esquina del claustro (Rohr 2005: 96).

Desde entonces, la conocida Casa de la Columna empezará a sufrir modificaciones que alterarán por completo su estructura original y la convertirán en una gran casa de vecindad, conventillo o con el apelativo peruano de callejón ${ }^{4}$. El claustro del antiguo noviciado fue convertido en una quinta de dos pisos. En los ejes de los pilares, paredes de madera separaban varias celdas estrechas y profundas. Cada celda tenía un ancho de 2,90 metros y una profundidad de 7,00 metros. La única forma de iluminación que entraba desde el semioscuro pasillo se daba a través de una ventana ubicada en el sobre luz de la puerta.

En el segundo piso, y por la disposición de los muros, el amplio claustro se convertía en un estrecho pasillo de quinta. Del ancho de 22 por 12,5 metros (275 metros cuadrados) solo quedaban 16 por 2,4 metros (38,4 metros cuadrados). Al mismo tiempo, la relación entre el 
área libre y el área construida fue empeorando considerablemente, puesto que el claustro colonial había tenido 600 metros cuadrados de área libre para 594 metros cuadrados de celdas, una relación de casi 1:1. En el nuevo edificio, 350 metros cuadrados de área libre servían para 1900 metros cuadrados de área construida. Además, el diseño se conformaba con una estructura con ventilación pobre. No más de un tercio de las habitaciones debían contar con una ventana o una teatina (Rohr 2005: 96).

Esta tugurización del claustro del noviciado, conocido desde entonces en el argot limeño como «Casa de la Columna», soportará mayores cambios arquitectónicos, cuyo único objetivo estará en función de que sea utilizado como una «vivienda» — sin los más mínimos requisitos de higiene y salubridad-y que genere los mayores recursos a los nuevos dueños, para lo cual no importaba el hacinamiento en que se hallaban los nuevos inquilinos, los mismos que irían en constante aumento.

A la vez, los dominicos seguirían vendiendo partes de su convento, como fue el caso de su antigua enfermería, en la cual se fundó el colegio de Santo Tomás de Aquino hacia 1892. Desde ese momento, el convento de Santo Domingo quedó reducido a su mínima expresión.

\section{Nuevos usos, nuevos problemas, nuevas evidencias}

Este cambio de uso, de un original asentamiento religioso utilizado para la formación de una élite conventual a una gran vivienda habitada por gente de diversos oficios y profesiones, por personas comerciantes, así como agricultores, poco a poco irá transformando las antiguas casas señoriales coloniales en viviendas compartidas y ocupadas por diferentes personas, de variada condición cultural y social, tal como puede verificarse en la Guía de domicilio de Lima (Fuentes 1863). Para mediados del siglo XIX, el convento de Santo Domingo ocupaba las calles de Matavilela y Veracruz, pero esta denominación será cambiada por la de "Jirón Lima». Justamente, en esta nueva calle, se encontrará el antiguo Claustro del Noviciado y, sobre sus antiguas edificaciones, se presentará una nueva configuración, tal como se daba el caso en otras casas. Este cambio morfológico fue anotado por el viajero americano George Squier: "La mayoría de las casas tienen patios con galerías abiertas que se extienden en torno de los cuatro lados. La planta baja, que da a la calle, está ocupada, por lo general, por tiendas y los restantes cuartos se dedican al almacenaje o son usados como caballerizas y cocinas, estas últimas a menudo adyacentes y a veces unidas» (1974: 23).

A su vez, esta nueva clase trabajadora, que cada vez más buscaba asentarse en aquella Lima semiurbana, trajo consigo la presencia de una nueva cultura material. Esta nueva clase proletaria, típica del siglo XIX, representada en la cotidianidad del mundo limeño, empezó a caracterizarse por el consumo de nuevos bienes, sobre todo, de origen europeo, tales como alimentos, vestidos, menaje de casa y, en general, todo objeto utilizado en la vida diaria. A la vez, muchos objetos empezaron a ser copiados y adulterados por los mismos y nuevos inquilinos. Nuevos hábitos de consumo alimenticios se darán gracias a la introducción de nuevas formas de etiqueta gastronómica importadas de Europa, así como a la aceptación y consumo de nuevos hábitos alcohó$\operatorname{licos}^{5}$, tal como quedó reflejado en los distintos registros arqueológicos excavados en la Casa de la Columna.

Sobre la base de los hallazgos encontrados durante las excavaciones efectuadas, podemos entender cómo transcurría la vida de los nuevos moradores y, también, las carencias que tenían. Esto atrajo la atención de las autoridades municipales, quienes, junto a los médicos, vieron desde entonces a las casas de vecindad como centros de insalubridad, en donde pululaban las enfermedades, y todo tipo de desgracia moral e inhumana (alcoholismo, vicios, enfermedades infecto contagiosas). Como prueba de este pequeño mundo, podemos destacar el interesante trabajo de Juan Portella, quien hacia 1908 realizó varias inspecciones oculares a diversas casas de vecindad, entre ellas, a la Casa de la Columna, en la cual describe el ambiente insalubre que existía; a la par, entrega interesantes fotografías de este lugar (Fig. 2). Cabe anotar que no solamente la Casa de la Columna sino otros sitios adyacentes atrajeron la atención de los llamados médicos higienistas, quienes inspeccionaron y dejaron sendos informes tal como el mencionado anteriormente. 


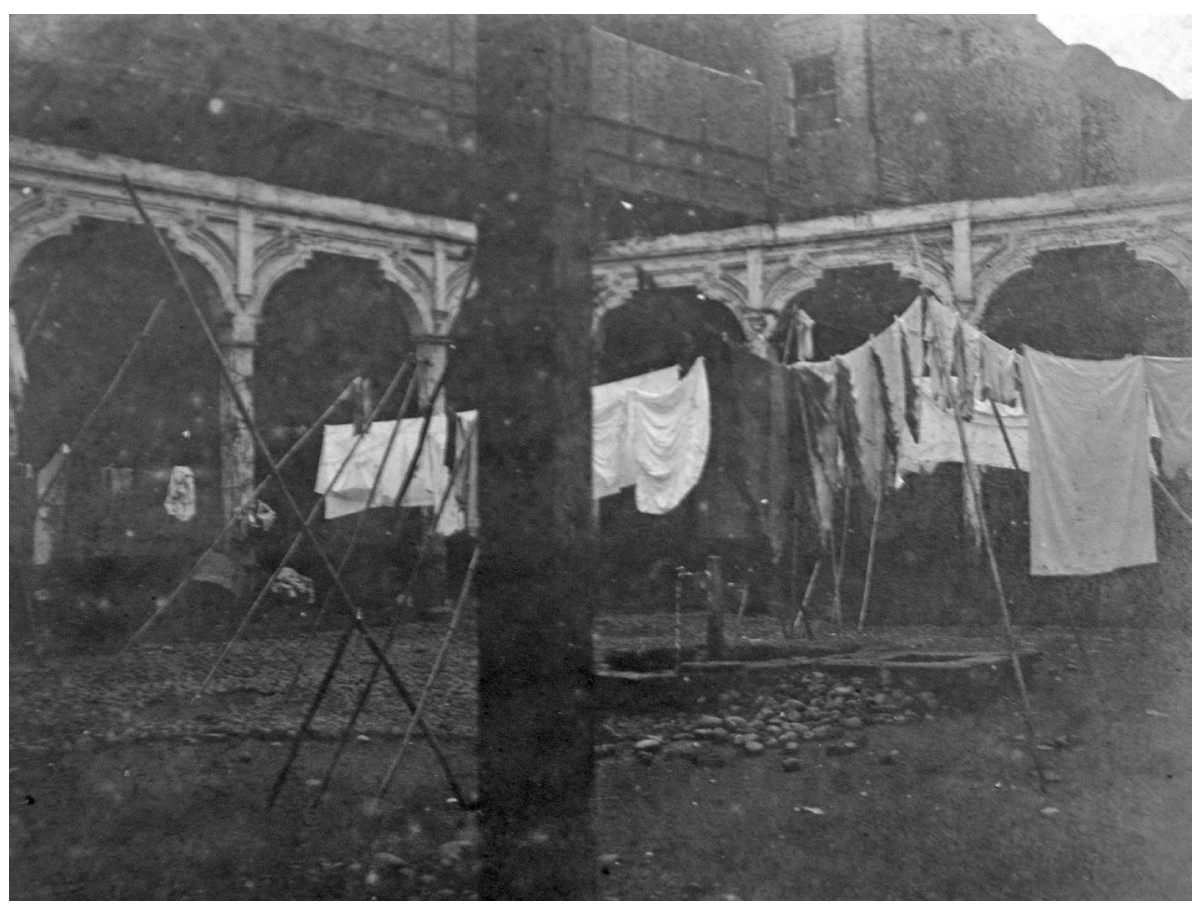

Figura 2. Interior de la Casa de la Columna a inicios del siglo XX (Fuente: Portella 1909).

Esta fue la caracterización de los inicios del siglo XX, aunque ya iniciada en el XIX: propietarios de muchas de las antiguas edificaciones conventuales y grandes casas residenciales del centro de Lima tuvieron la intención y el interés de convertir estos sitios originalmente construidos para otros fines en conjuntos de alto rendimiento económico, sin considerar la calidad del mismo, su higiene y salubridad. Las edificaciones específicamente conventuales eran bienvenidas, pero no para rezar, sino para ahorrar costos de construcción. Estas nuevas viviendas, nacidas de una "celda monacal», ahora eran denominadas como "callejón de cuartos», y se caracterizaban por tener una pieza con una alacena en el muro, una cocina con fogón y sus pisos eran de tierra apisonada ( $c f$. Harth-Terré y Márquez Abanto 1962: 161).

Del material obtenido, podemos destacar, en primer lugar, la cerámica tanto colonial como republicana, la cual es la más representativa y nos sirve para obtener información sobre los motivos decorativos y diversas formas utilizadas, sean escudillas, cuencos, tazas, copas y demás menaje de casa. No queremos entrar en detalles sobre los estilos ceramográficos coloniales, pues creemos que aún no tenemos una secuencia maestra que nos sirva de guía para clasificar a la cerámica peruana o la limeña de los siglos XVI, XVII y XVIII. Para no caer en la letanía de repetir los estilos foráneos y superponerlos a la fuerza a nuestro material, tales como Panamá Polícromo, Panamá Blanco sobre Azul, entre otros, simplemente los dividiremos de manera más fácil, pero sí claramente definidos y caracterizados como cerámica colonial y cerámica republicana.

De la cerámica colonial, se caracterizan las llamadas botijas, peruleras y toda su diversidad de formas y subformas. Asimismo, existen otras variedades de recipientes, tales como escudillas, copas, tazas, cuencos, jarras, etc. (Zuzunegui 1965). En cuanto a los colores, predomina el color blanco; las otras muestras son diversos fragmentos de color azul lapizlázuli, mientras que otras presentan colores verde, azul y violeta (Fig. 3).

En cuanto a la cerámica republicana, esta presenta como motivos decorativos soldados de caballería, con fondos de castillos medievales. Asimismo, abundan los motivos de torres con almenados, arcos ojivales y arcos de medio punto. Otro motivo a destacar es el de los paisajes bucólicos, 


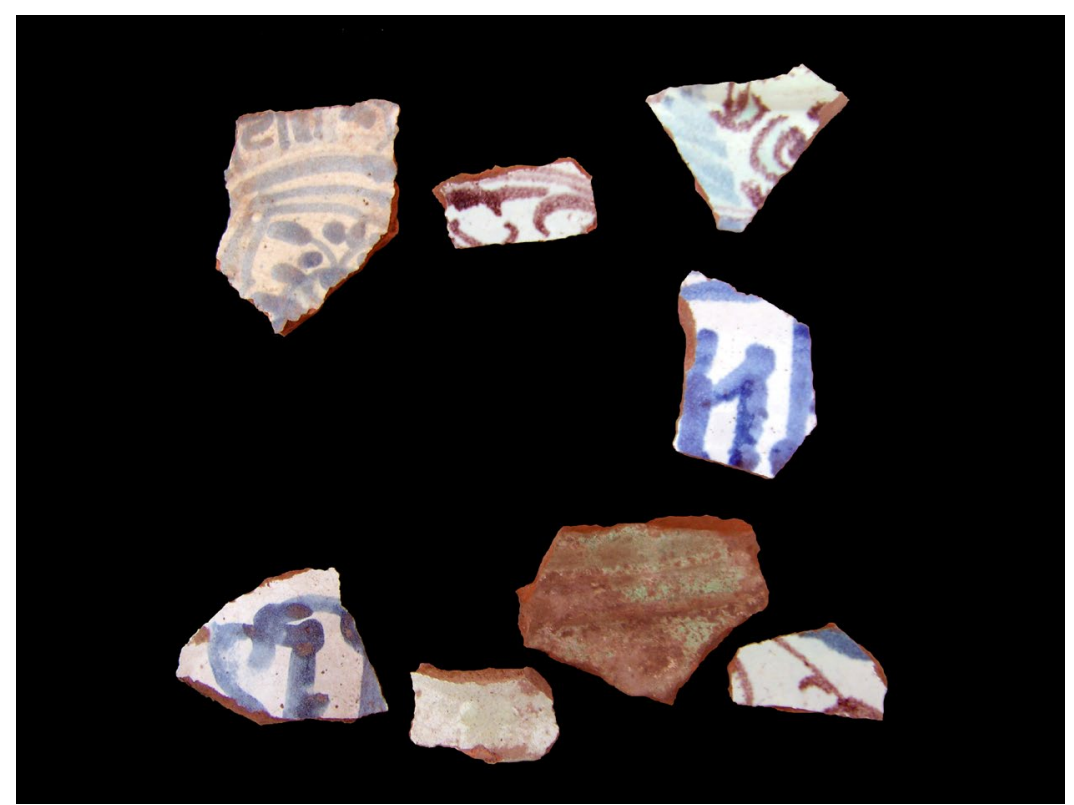

Figura 3. Cerámica colonial, de estilo Panamá (Foto por A. Coello).

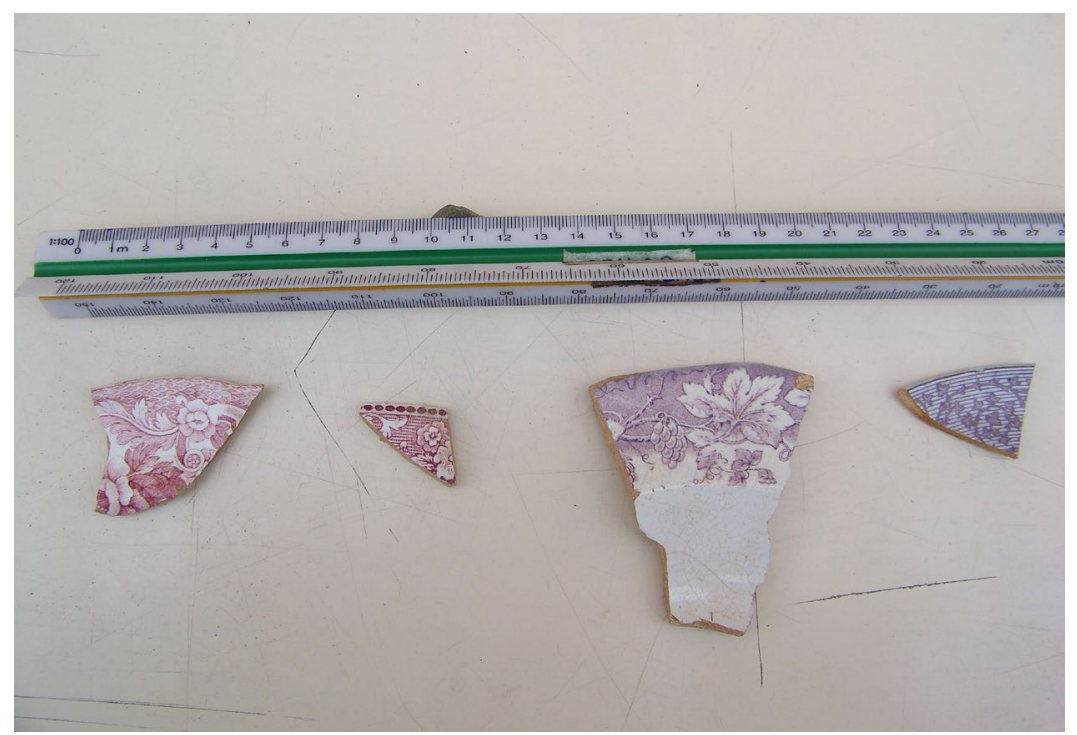

Figura 4. Diversas muestras de cerámica republicana (Foto por A. Coello).

asociados con escenas de hombres y mujeres en actos románticos. Esta muestra, por lo general, mantiene colores blancos en los fondos, y azules y rojos en los bordes de las tazas y platos (Fig. 4). Cabe mencionar los fragmentos de botellas medicinales, tales como L'eau de Pin y diversos frascos de perfumes (Fig. 5).

Una mención aparte merece la de los restos de botellas de bebidas alcohólicas (Fig. 6), las cuales fueron consumidas en grandes cantidades. Tuvimos la suerte de que, durante las excavaciones, hallásemos algunas botellas enteras. Estas fueron identificadas gracias a la ayuda de fuentes históricas publicadas e inéditas ${ }^{6}$. 


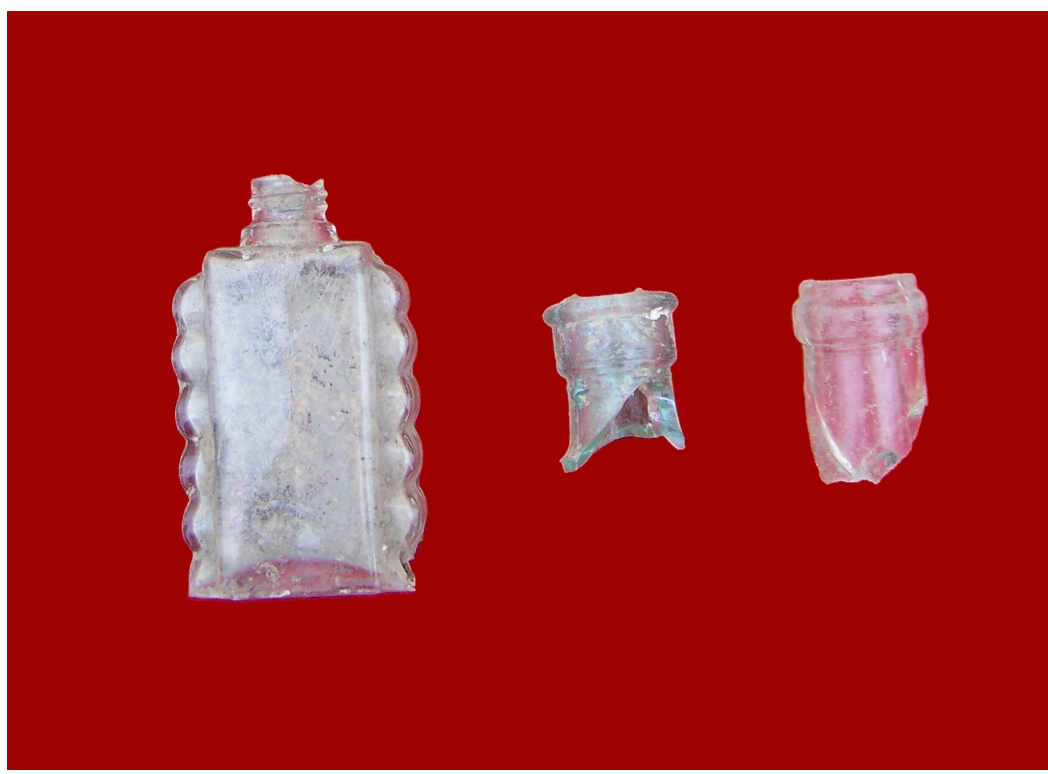

Figura 5. Diversas muestras de frascos de vidrio (Foto por A. Coello).

Otro componente arqueológico a mencionar es el material óseo animal, el cual apareció solamente en el segundo patio. Creemos que estos restos son el resultado de la alimentación efectuada por el destacamento policial que habitó la Casa de la Columna durante las décadas de 1830 y 1840. A la fecha, no hemos obtenido los resultados del análisis de este material por carencia de fondos investigativos, pero creemos que, cuando los obtengamos, estos nos brindarán nuevas luces para comprender cómo fueron utilizados y si los consumieron, así como el debido tratamiento que tuvieron.

\section{Sobre las excavaciones}

Durante el proceso de excavación, se abrieron en total 19 unidades divididas de la siguiente manera: 15 en el primer patio y cuatro en el segundo patio. No entraremos en detalle específico sobre el proceso de excavación arqueológica, pues es muy extenso y a veces cae en el tecnicismo únicamente entendible quizás por los propios arqueólogos. Por tal razón, presentaremos los rasgos más importantes de algunas unidades y nuestras hipótesis de trabajo sobre lo hallado durante la excavación (Fig. 7).

Comenzaremos por el segundo patio, que es el menos denso y de mayor importancia, por los hallazgos, para entender la historia de la Casa de la Columna. En el segundo patio, se abrieron cuatro unidades de excavación: tres de ellas se ubicaron en medio del patio, mientras que una se instaló a los pies de la portada que daba acceso al mismo (Fig. 8). Esta unidad, la 12, tenía por finalidad hallar los cimientos de dicha portada, y ubicar los posibles restos de cañerías o albañales colapsados que dańaban con humedad a dicha portada.

Las Unidades 13 y 14 nos dieron importantes hallazgos, tales como abundante material óseo animal, mezclado con material cultural (restos de menaje y vajilla de cocina en general) ${ }^{7}$. Esta unidad reveló gran cantidad de desechos orgánicos e inorgánicos (restos de basura), los cuales fueron compactados con el tránsito continuo de los habitantes. Esto se acentuaba con el vertido del agua que se empleaba para limpiar momentáneamente este patio. Estas evidencias son claramente representativas de la vida cotidiana del siglo XIX, cuando una parte del antiguo claustro original se convirtió en un cuartel de policía y la otra parte en recintos habitacionales. 


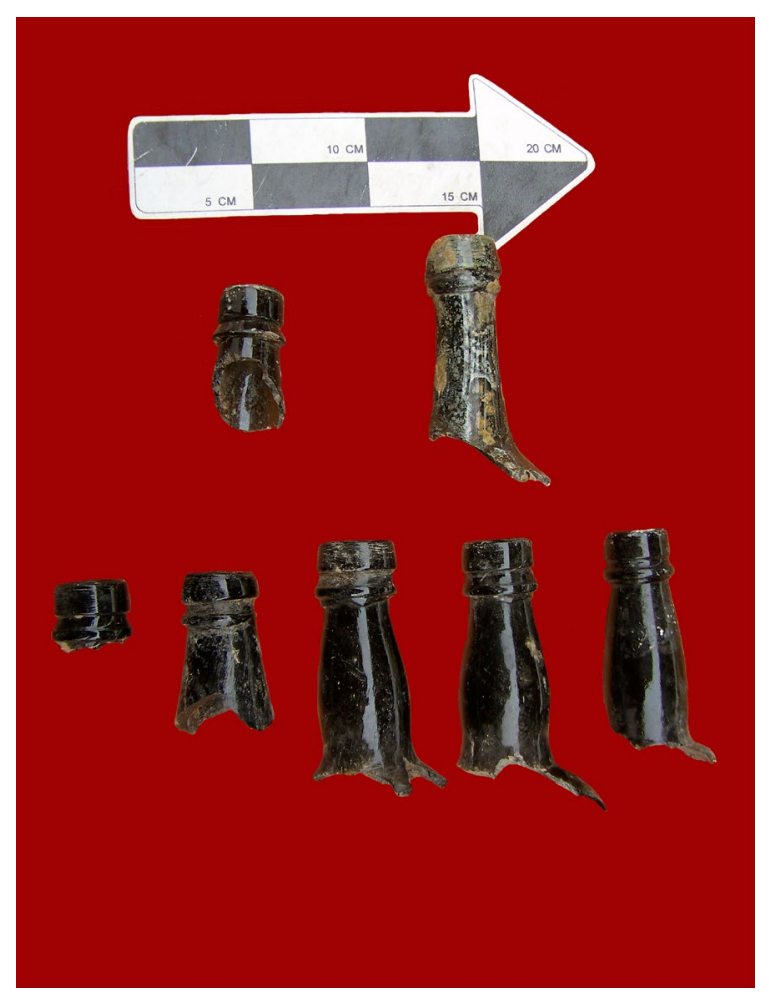

Figura 6. Fragmentos de botella de bebidas alcohólicas (Foto por A. Coello).

Por último, la Unidad 15 también nos mostró abundante evidencia de fragmentería de cerámica colonial asociada a un piso de ladrillo rojo, que creemos formó parte del convento original del siglo XVII (Fig. 9). Estos restos de piso también los hallamos en el primer patio, en las Unidades $1,2,3,4$ y 16 . El piso presenta un tramado conocido como espina de pez y colindante al mismo, un sardinel formado por cantos rodados. Creemos que dichos elementos constructivos formaban parte de un claustro; los cantos rodados eran el límite que daba paso al jardín o patio propiamente dicho, mientras que el piso de ladrillos rojos conformaba la galería-corredor del claustro.

Por otra parte, en el primer patio, se abrieron 15 unidades. De estas, las 8, 9 y 10 se emplazaron en el medio del claustro o actual patio; las Unidades 2, 4, 5, 6, 7 y 16 se distribuyeron a lo largo de la galería-corredor que circunda al claustro, mientras que, en el zaguán de entrada que comunica a la casa con el jirón Conde de Superunda, se colocaron las restantes.

En las Unidades 8, 9 y 10, colocadas en el medio del patio, se tenía como finalidad hallar los posibles restos de la pileta que existió en este antiguo claustro del noviciado, así como ver las huellas de las antiguas canaletas que distribuían agua y que fueran señaladas en el plano de Meléndez (1681). Sin embargo, nuestros hallazgos no fueron tan alentadores, puesto que no encontramos lo deseado, aunque sí restos de antiguos ductos de cańerías de plomo, los cuales sabemos que fueron colocados en la ciudad de Lima a mediados de 1850, durante el gobierno de Ramón Castilla (Regal 1967). Dichos ductos estaban parcialmente destruidos.

Las unidades colocadas en la galería-corredor se instalaron, a su vez, colindantes a las columnas que forman la arquería. Estas servirían para identificar y explicar los diferentes momentos constructivos que soportó el convento. El común denominador en todas estas calas exploratorias fue que se halló la misma secuencia estratigráfica. Comenzaremos por explicar el proceso, de arriba hacia abajo, seńalando que, luego de extraer el piso de cemento que representa el nivel actual de uso, se hallaron dos niveles, un apisonado informal con restos de caliche y tierra arcillosa semicompacta. 


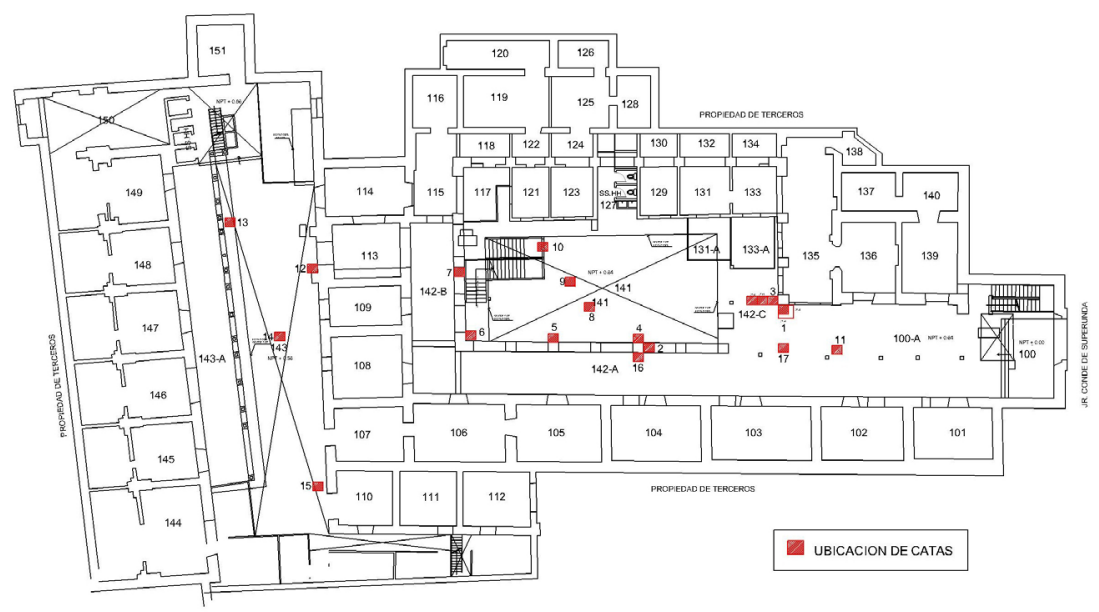

Figura 7. Ubicación de las unidades de excavación.

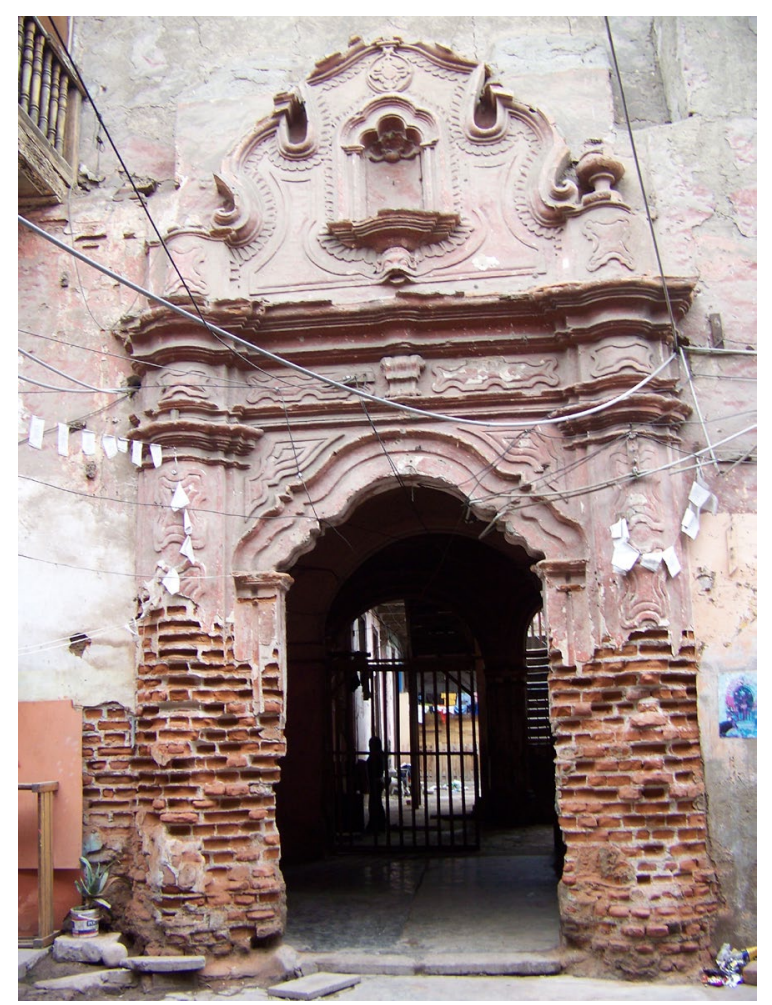

Figura 8. Portada de la Casa de la Columna en su estado actual (Foto por A. Coello). 


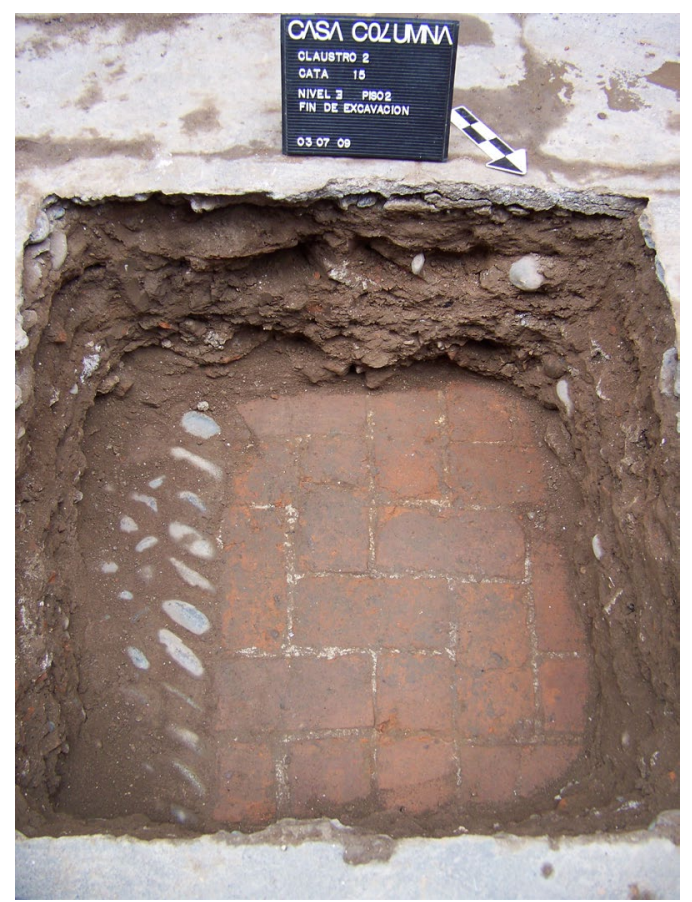

Figura 9. Piso de la unidad 15 (Foto por A. Coello).

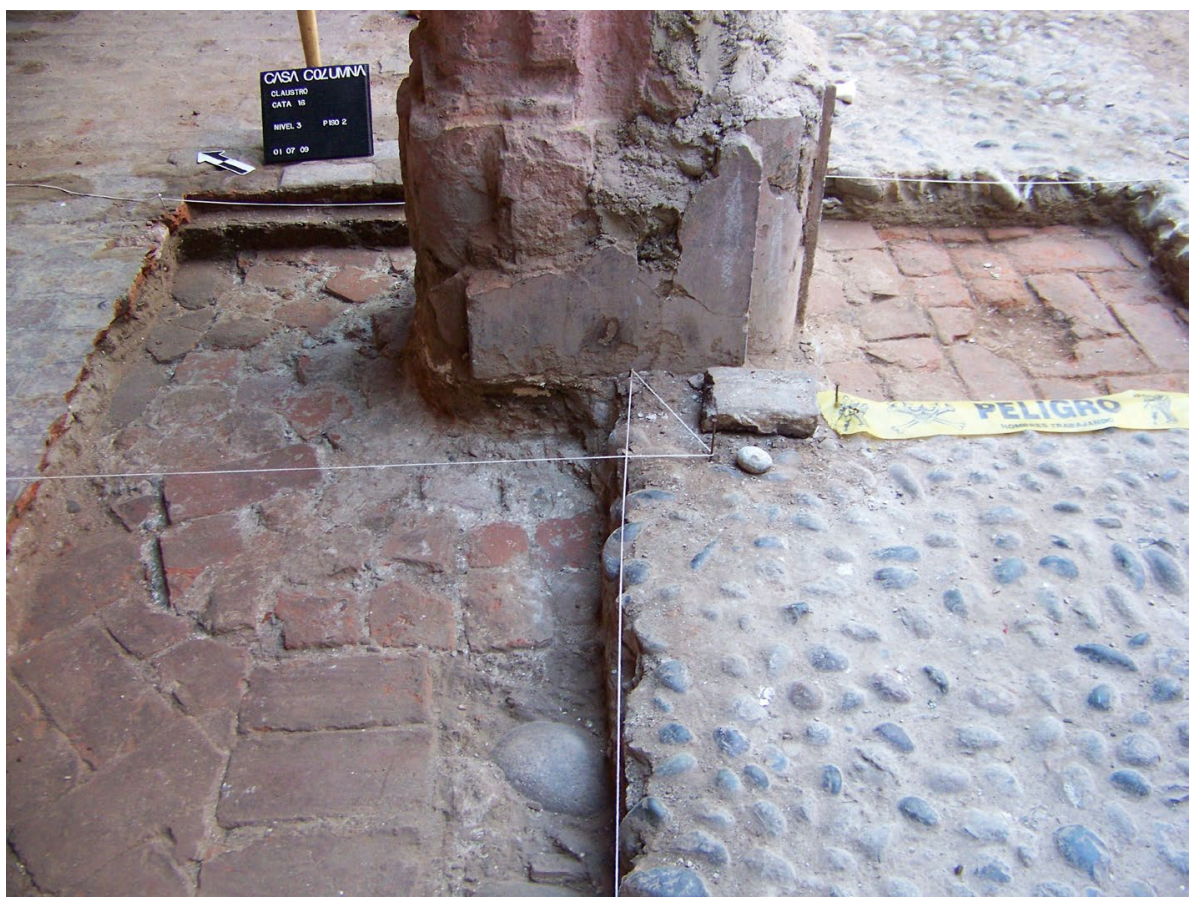

Figura 10. Piso de la unidad 16 (Foto por A. Coello). 


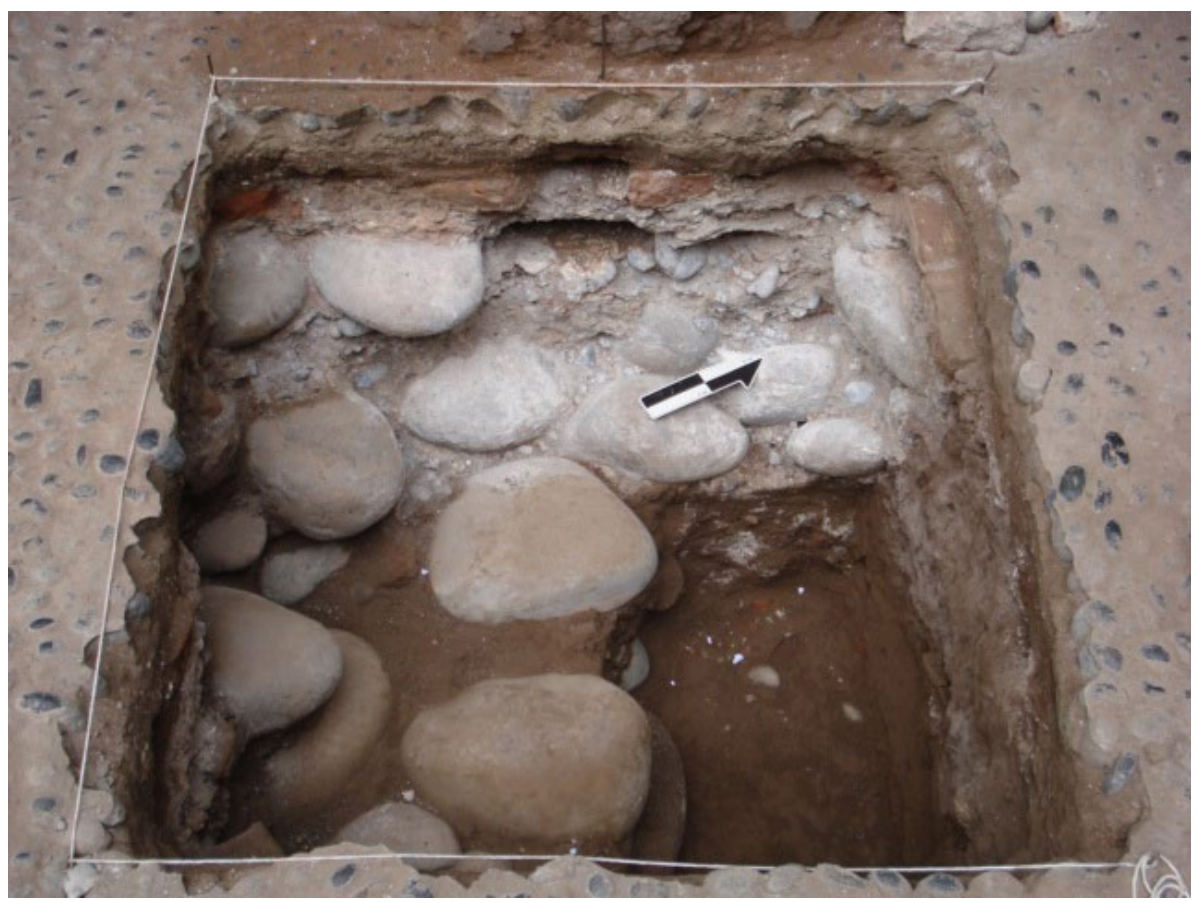

Figura 11. Fin de excavación de la unidad 5 (Foto por A. Coello).

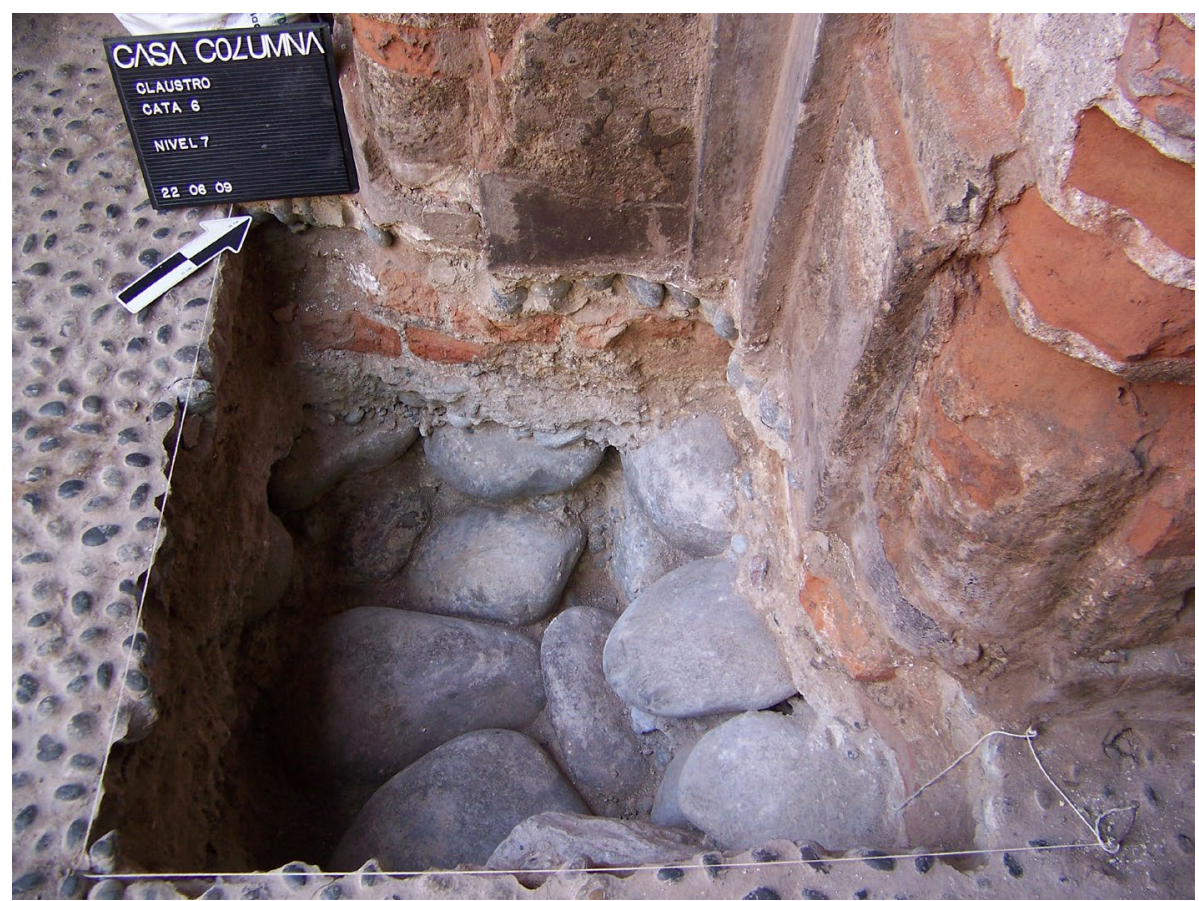

Figura 12. Fin de excavación de la unidad 6 (Foto por A. Coello). 
Por debajo de esta última, se halló el ya mencionado piso de ladrillo rojo, el mismo que apareció en el segundo patio. Debemos indicar que, en algunas unidades, apareció íntegramente el piso, mientras que en otras apareció solo parcialmente. Como ejemplo, se puede ver el piso de las Unidades 2, 4 y 16, el mismo que circundaba a la columna (Fig.10). En las unidades en las que apareció parcialmente el piso, continuamos descendiendo y hallamos sucesivas capas formadas por grandes cantos rodados. Creemos que estas serían parte de los cimientos del claustro. No debemos olvidar que, en sus inicios, el convento de Santo Domingo colindaba con el río Rímac, lugar en donde se pudo extraer fácilmente este material constructivo; los cantos rodados están mezclados con calicanto, lo cual los hace más compactos y resistentes al paso de los años (Figs. 11 y 12).

En cuanto a la técnica constructiva encontrada por nosotros, existen datos históricos, publicados por Harth-Terré (1950) y Harth-Terré y Márquez Abanto (1962), sobre las técnicas constructivas, el sistema de cimientos y los pisos, que son semejantes a lo hallado por nosotros.

\section{Agradecimientos}

La presente investigación formó parte de un proyecto de restauración, que contó con el financiamiento de la World Monuments Fund, y la asesoría técnica de la Escuela Taller de Lima y el CIDAP en convenio con la Sociedad de Beneficencia Pública de Lima, actual propietaria. Asimismo, en la dirección técnica arquitectónica, debemos agradecer a las arquitectas Iraida Carbajal y Violeta Paliza, y al especialista Francisco Quispetierra, así como al equipo de arqueólogos, integrado por los especialistas Giannina Bardales, Henry Tantaleán y César Trigoso. A todos ellos queremos expresarles nuestro sincero y eterno agradecimiento por su colaboración, profesionalismo y entrega desinteresada.

\section{Notas}

${ }^{1}$ Hemos utilizado la equivalencia de la vara castellana en relación de 772 milímetros.

${ }^{2}$ Refiere a la cesión perpetua o por mucho tiempo, de bienes raíces, mediante un canon anuo que se paga al cedente, quien conserva el dominio directo (Real Academia Espańola 1980: 1271).

${ }^{3}$ Archivo Histórico del Convento de Santo Domingo.

${ }^{4}$ Para entender estas definiciones y comparar el caso chileno con el peruano, se puede consultar a Urbina Carrasco (2002) y Ramón (1998).

${ }^{5}$ Nos referimos de manera específica a los restos hallados de nuevas bebidas alcohólicas que no se consumían en Lima, tales como Cinzano, Vermouth, Cerveza Guiness, Ginebra y otras.

${ }^{6}$ En el Archivo Histórico de la Municipalidad de Lima, en la sección «Higiene y salubridad», hallamos varios expedientes sobre la adulteración y consumo de diversos licores que se comercializaban en la segunda mitad de la Lima del XIX.

${ }^{7}$ En este segundo patio, se hallaron restos y, en otros casos, botellas enteras de bebidas alcohólicas. 


\section{REFERENCIAS}

Angulo, D.

1939 El terremoto del año 1687, Revista del Archivo Nacional, tomo XII, entrega 1, enero-junio, 1-70.

Cobo, B.

1882 Historia de la fundación de Lima [edición de M. González de la Rosa], Imprenta Liberal, Lima.

[1613]

Fuentes, M. A.

1863 Guía de domicilio de Lima, imprenta del autor, Lima.

Harth-Terré, E.

1950 Cómo eran las casas en Lima en el siglo XVI, Mar del Sur. Revista Peruana de Cultura 11(10), 17-29.

Harth-Terré, E. y A. Márquez Abanto

1962 Historia de la casa urbana virreinal en Lima, Revista del Archivo Nacional del Perú, XXVIII, 107-210.

\section{Huertas L. e I. Flores}

1981 Investigación arqueológica- histórica de la Casa de Osambela, Instituto Nacional de Cultura/Centro de Investigación y Restauración de Bienes Monumentales, Lima.

Luna, P.

2002 Conventos, monasterios y propiedad urbana en Lima, siglo XIX: El caso de la Buenamuerte, Fronteras de la Historia 7, 141-163.

Meléndez, J. de

1681 Tesoros verdaderos de las Indias, tomos I, II y III, Imprenta de Nicolás Angel Tinassio, Roma.

Radiguet, $M$.

1971 Lima y la sociedad peruana, Biblioteca Nacional del Perú, Lima.

Ramón, G.

1998 La muralla y los callejones, Sidea, Lima.

Real Academia Española

1980 Diccionario Enciclopédico Ilustrado de la Lengua Española, tomo II, Sopena, Barcelona.

Regal, A.

1967 Castilla Constructor, Instituto Libertador Ramón Castilla, Banco de Crédito del Perú, Lima.

Rohr, D.

2005 Ciudad y bienes raíces. Las órdenes religiosas en Lima. El Convento Dominico (1534-1890), Urbes 2 (2), 77-101.

Squier, G.

1974 Un viaje por tierras incaicas. Crónica de una expedición arqueológica (1863-1865), Editorial Los Amigos del Libro, La Paz.

Ulloa, A. de y J. Juan

1748 Relacion historica del viage a la America Meridional hecho de orden de S. Mag. para medir algunos grados de

[1713- meridiano terrestre y venir por ellos en conocimiento de la verdadera figura y magnitud de la tierra, con otras

1776 ] observaciones astronomicas y phisicas, tomo III (publicado por A. Marin), Imprenta Real, Madrid.

Urbina Carrasco, $\mathrm{X}$.

2002 Los conventillos de Valparaíso 1880-1920, Ediciones de la Universidad Católica de Valparaíso, Valparaíso.

Walker, Ch.

2012 Colonialismo en ruinas. Lima frente al terremoto y tsunami de 1746, Instituto de Estudios Peruanos, Lima. https://doi.org/10.4000/books.ifea.6620

Zuzunegui, A.

1965 Recipientes cerámicos utilizados en el comercio de Indias, Boletin de Americanistas 19, 21-38. 